\title{
Advanced modulation coding schemes for an optical transceiver systems-based OWC communication channel model
}

\author{
Hazem M. El-Hageen ${ }^{1}$, Aadel M. Alatwi ${ }^{2}$, Ahmed Nabih Zaki Rashed ${ }^{3}$ \\ ${ }^{1}$ Egyptian Nuclear \& Radiological Regulatory Authority, Cairo, Egypt \\ ${ }^{1,2}$ Electrical Engineering Department, Faculty of Engineering, University of Tabuk, Tabuk, Saudi Arabia \\ ${ }^{3}$ Electronics and Electrical Communications Engineering Department, Faculty of Electronic Engineering, Menoufia \\ University, Egypt
}

\begin{abstract}
Article Info
Article history:

Received Aug 14, 2020

Revised Nov 21, 2020

Accepted Dec 5, 2020

Keywords:

Modulation coding schemes

Optical receiver

Optical transmitter

ABSTRACT

This paper examines advanced modulation coding schemes for an optical transceiver system-based optical wireless communication (OWC) channel model. These modulation techniquesinclude On-Off keying and return to zero (RZ)/non-return to zero (NRZ) coding. The signal power level against time and frequency spectral variations are measured. The max. Q factor and min. bit error rate (BER) are estimated and clarified for each modulation code scheme by using an optisystem simulation model. Transmission bit rates of up to $40 \mathrm{~Gb} / \mathrm{s}$ can be achieved for possible distances up to $500 \mathrm{~km}$ with acceptable $\mathrm{Q}$ factor. The received power and max. Q factor are measured and clarified with OWC distance variations. The On-Off keying modulation code scheme resulted in better performance than the other modulation code schemes did.
\end{abstract}

Owc channel

This is an open access article under the CC BY-SA license.

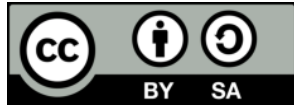

\section{Corresponding Author:}

Ahmed Nabih Zaki Rashed

Electronics and Electrical Communications Engineering Department

Faculty of Electronic Engineering, Menoufia University

Gamal Abd El-Nasir, Qism Shebeen El-Kom, Shibin el Kom, Menofia, Egypt

Email: ahmed_733@yahoo.com

\section{INTRODUCTION}

Free space optics (FSO) communication is a growing technology to handle high data rate and it has very large information handling capacity [1-4]. FSO communication systems are presented as an available alternative to the fiber optics technology which is capable of full duplex transmission of data, voice and video. Even though light can be competently inserted into fiber cables to route the light information [5-9], there are various applications where only the free space between the transmitter and receiver is the only available means to establish a communication link. This free space technique needs only a clear line of sight path between the transmitter and the remote receiver [10-14].

The demands for solutions for traffic problem such as accidents, jams and environmental impact are increasing. Heavy economical losses are caused by traffic congestion apart from inconvenience to users [15-19]. Visible light communication systems have multiple benefits [20-25]. Indoor wireless communication used two major transmission technologies, they are RF and Optical Wireless Communication (OWC) systems. While the requirement of line of sight link, atmospheric absorption, scattering and scintillation can be thought of as drawbacks of the OWC system, it becomes advantageous in the indoor from one room to another room or cell based links without interference from each other [26-34]. Also, OWC has many advantages such as it is inexpensive, low power consumption, quickly-deployable and provides security over RF communication. Infrared (IR) to Ultraviolet (UV) including visible light are used in OWC [35], this wide 
range and unlicensed bandwidth make OWC system has potential to deliver several hundreds of Mbps data rate [36]. Many studies show that OWC able to transmit at data rates up to 25 Gbps in indoor systems [37-40]. The first and the foremost limiting factor for achieving high data rate in indoor OWC is the limited modulation bandwidth of light sources. There are various modulation techniques have been used in indoor OWC System [41-45]. The using of either depends on the intended application and channel configuration. For example, OOK (On-Off Keying) and PPM (Pulse Position Modulation) are favored for high power effectiveness. Pulse interval modulation (PIM) procedures are utilized presumed for its intrinsic synchronization pulse. Differential PPM (DPPM), Differential Amplitude PPM (DAPPM) Multilevel Digital PIM (MDPIM), Digital PIM (DPIM) and Dual Header PPM (DH-PIM) are the substitutes to improve power efficiency, bandwidth efficiency and speed throughput. Trellis coded PPM (TC_PPM) enhances the execution of the PPM on multipath channels. Multiple PPM (MPPM) minimizes the impact of multipath scattering.OOK is a most simplest modulation technique for intensity modulation/direct detection (IM/DD), which represents one bit for an optical pulse and a bit zero for the absence of an optical pulse. The paper presents a simulation of different OOK modulation code schemes with estimation of their parameters which can be used to easy compare among the proposed schemes.

\section{RESEARCH METHOD}

This study used advanced modulation schemes such as On-Off keying, NRZ, and RZ. On-Off keying represents a binary data stream in the absence of a carrier signal. Bit sequence user-defined sequence generators were used to generate a stream sequence of bits as 10-bit sequences of 0101101110. The transmitter consists of two electrical units/light sources with two data user-defined generators and Gaussian/NRZ pulse generators. The sequence stream bits were encoded through pulse code generators. The technical specifications for the light optical sources included frequency $=193.1 \mathrm{THz}$ and power $=0 \mathrm{dBm}$. After encoding, the electrical/light signals then needed to be modulated through $\mathrm{LiNbO}_{3} \mathrm{Mach}$ Zehnder modulators using the following technical parameters: extinction ratio $=20 \mathrm{~dB}$, switching bias/switching RF voltages of 4 $\mathrm{V} / 2 \mathrm{~V}$ respectively, and insertion losses $=5 \mathrm{~dB}$. Figure 1 shows the optical transceiver system based OWC communication system model.

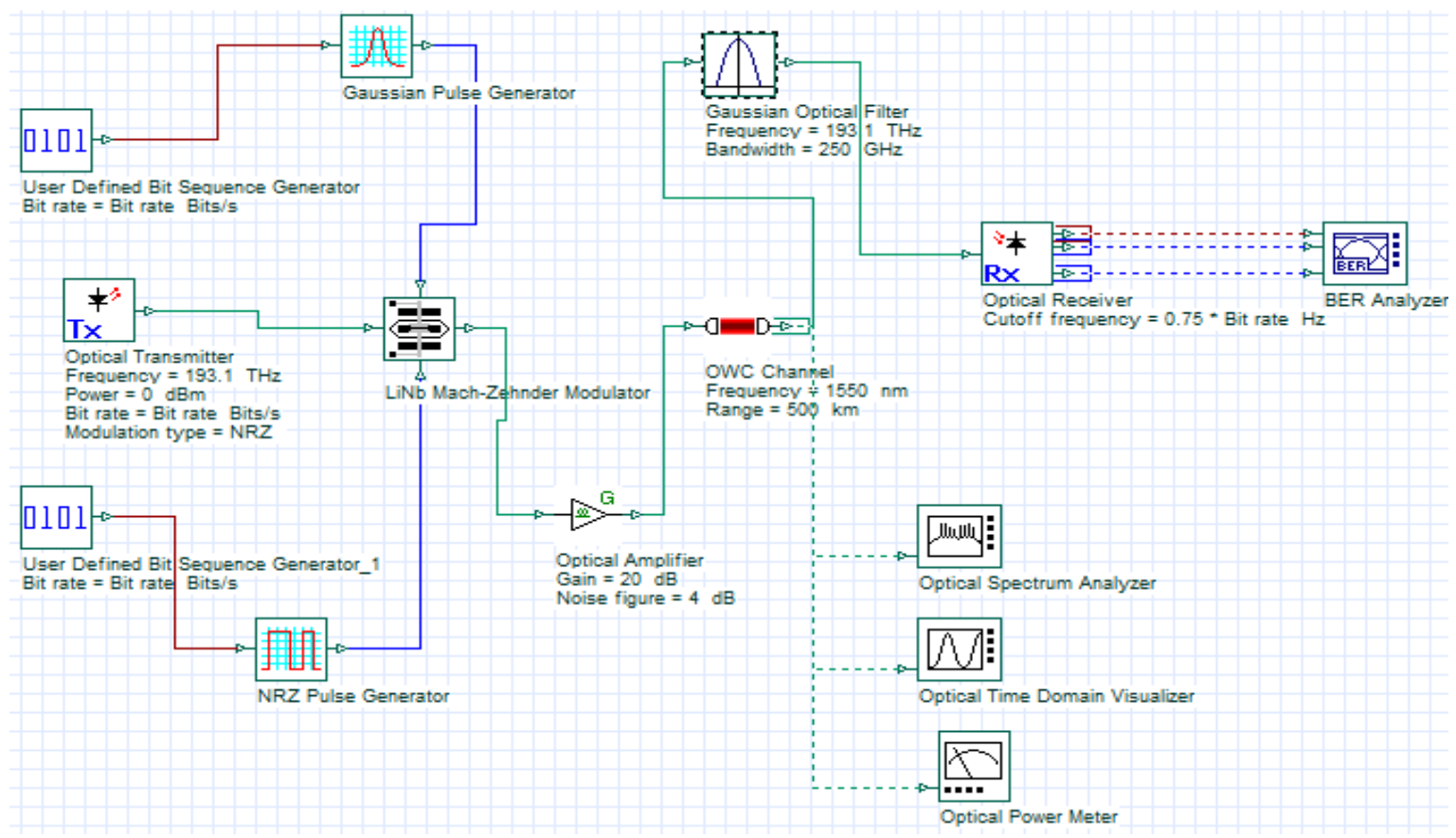

Figure 1. Optical transceiver system based OWC communication channel model

The modulated signal was then amplified with light amplifiers to compensate for losses before it was injected into the OWC channel with a distance of $500 \mathrm{~km}$. The signal was filtered through Gaussian light filters to eliminate all the ripples from the modulated original signal. The Gaussian light filter used the following technical variables: bandwidth $=250 \mathrm{GHz}$, frequency $=193.1 \mathrm{THz}$, loss $=0 \mathrm{~dB}$, filter order $=1$, and 
filter modulation depth $=100 \mathrm{~dB}$. Then, the filtered signal was converted from light signal form to electrical signal form through the light receiver side. All the clarified measurement devices measured the max. Q factor/min. bit error rate, the max. signal power/min. noise power, and the total received power after the light detectors (optical receiver side). All simulation results are shown based on the variables in Table 1.

Table 1. Parameters used in this proposed work

\begin{tabular}{lll}
\hline \multicolumn{3}{c}{ Transmitter technical specifications } \\
\hline \multirow{3}{*}{ Optical } & Frequency & $193.1 \mathrm{THz}$ \\
Tx. & Power & $0 \mathrm{dBm}$ \\
& Extinction ratio & $10 \mathrm{dBm}$ \\
& Linewidth & $10 \mathrm{MHz}$
\end{tabular}

\begin{tabular}{ll}
\multicolumn{1}{r}{ OWC channel technical specification } \\
Frequency & $1550 \mathrm{~nm}$ \\
Range & $500 \mathrm{~km}$ \\
Data rate & $40 \mathrm{~Gb} / \mathrm{s}$ \\
Tx. Aperture diameter & $15 \mathrm{~cm}$ \\
Rx. Aperture diameter & $15 \mathrm{~cm}$ \\
Tx. gain & $\mathrm{dB} 0$ \\
Rx. gain & $0 \mathrm{~dB}$ \\
Tx. Optics efficiency & 1 \\
Rx. Optics efficiency & 1 \\
Tx. Pointing error & $0 \mu \mathrm{rad}$ \\
Rx. Pointing error & $0 \mu \mathrm{rad}$ \\
Attenuation & $0 \mathrm{~dB} / \mathrm{km}$
\end{tabular}

Receiver technical specifications

\begin{tabular}{ll} 
Photodetector & PIN \\
Gain & 3 \\
Ionization factor & 0.9 \\
Dark current & $10 \mathrm{nA}$ \\
Responsitivity & $\mathrm{A} / \mathrm{W} 1$ \\
Insertion loss & $0 \mathrm{~dB}$ \\
Internal filter order & 1 \\
\hline
\end{tabular}

\section{RESULTS AND DISCUSSION}

Figures 2-4 show the max. Q factor with min. BER values for the various modulation coding schemes at a 500-km transmission distance. Figure 2 clarifies the max. Q factor was 6.45 and the min. BER was $5.08 \times 10^{-11}$ when using the NRZ modulation coding scheme. Figure 3 outlines the max. Q factor was 5.146 and the min. BER was $1.29 \times 10^{-7}$ when using the RZ modulation coding scheme. Figure 4 shows that the max. Q factor was 7.42 and the min. BER was $5.76 \times 10^{-14}$ when using the On-Off keying modulation coding scheme.
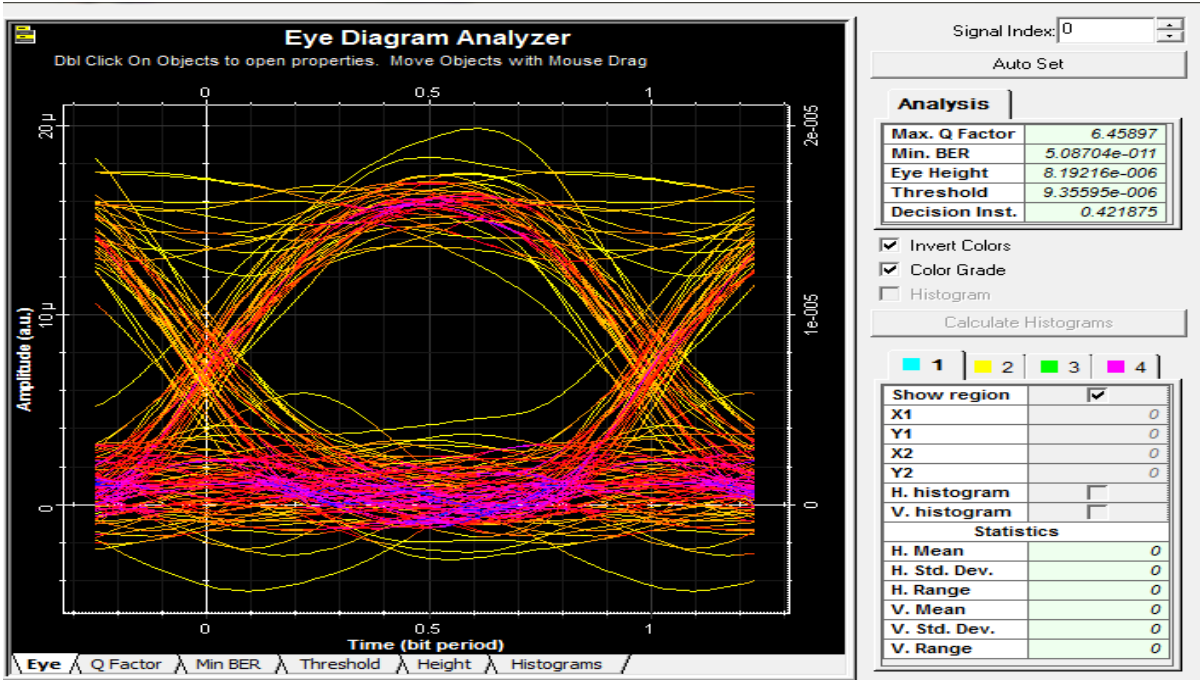

Figure 2. Max. Q factor with min. BER values by using NRZ modulation coding scheme based OWC channel (500 km distance) for optical transceiver systems

Advanced modulation coding schemes for an optical transceiver systems-based... (Hazem M. El-Hageen) 

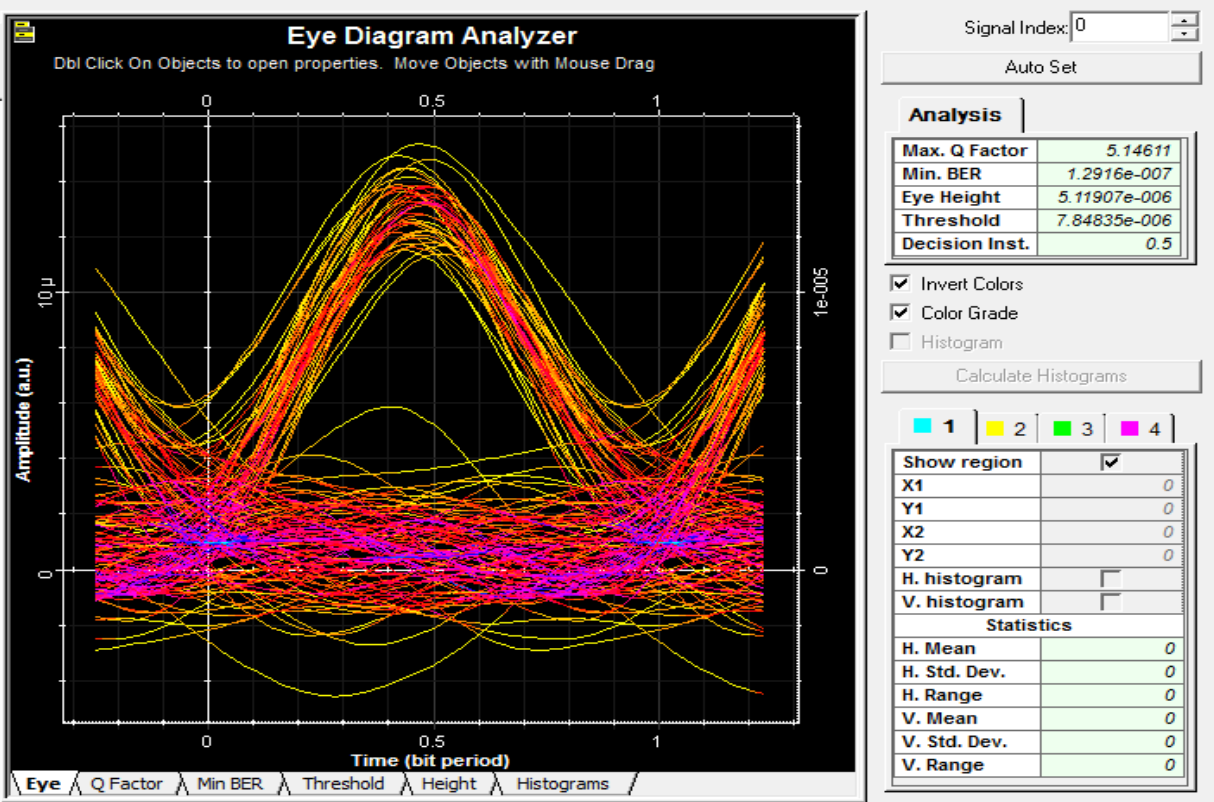

Figure 3. Max. Q factor with min. BER values by using RZ modulation coding scheme based OWC channel (500 km distance) for optical transceiver systems
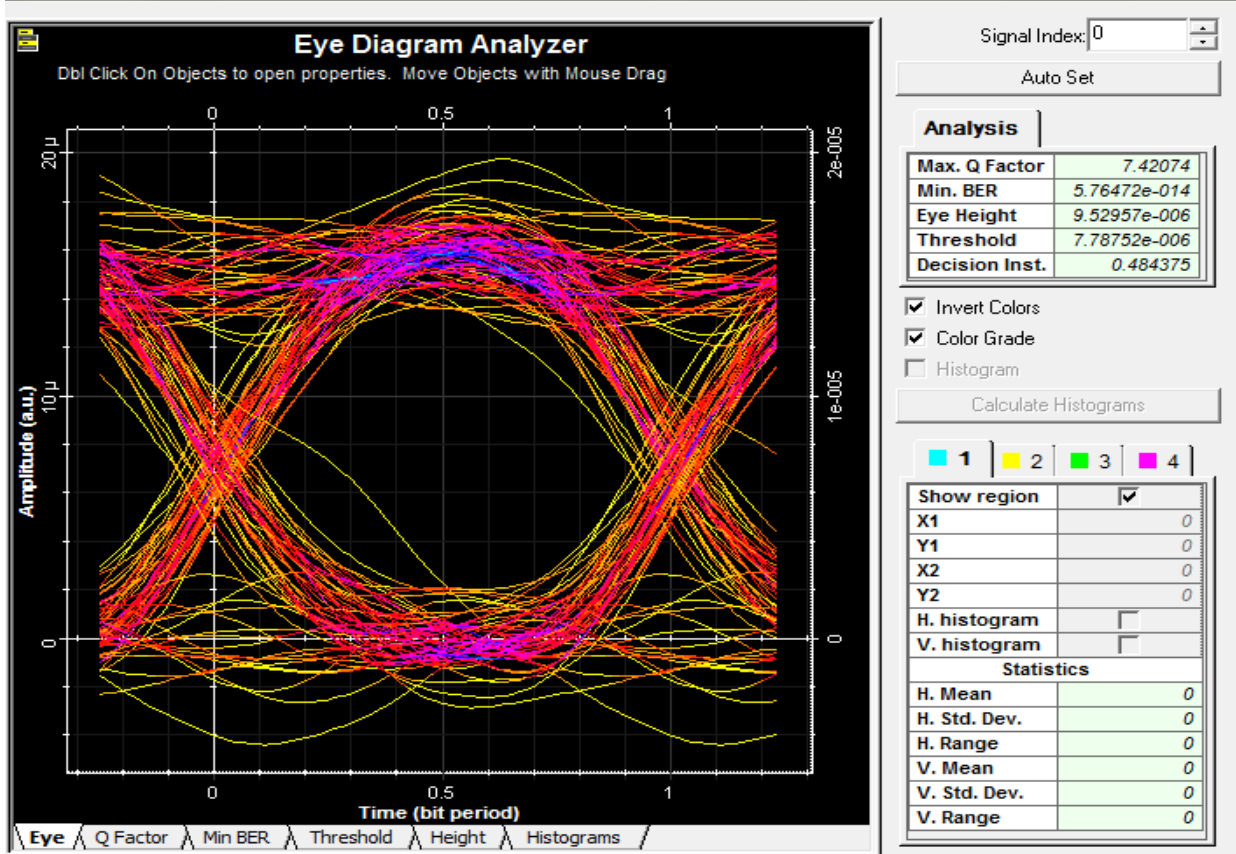

Figure. 4 Max. Q factor with min. BER values by using On Off keying modulation coding scheme based OWC channel (500 km distance) for optical transceiver systems

Figures 5-7 show the max. signal power/min. noise power when using the various modulation coding schemes at a 500-km distance. Figure 5 clarifies he max. signal power/min. noise power values were $-22.67 \mathrm{dBm}$ and $-103.682 \mathrm{dBm}$ when using the NRZ modulation coding scheme. Figure 6 shows the max. signal power/min. noise power values using the RZ modulation coding scheme were $-25.43 \mathrm{dBm}$ and $-103.551 \mathrm{dBm}$. As Figure 7 shows, the max. signal power/min. noise power values were $-19.09 \mathrm{dBm}$ and $-103.853 \mathrm{dBm}$ for the On-Off keying modulation coding scheme. 

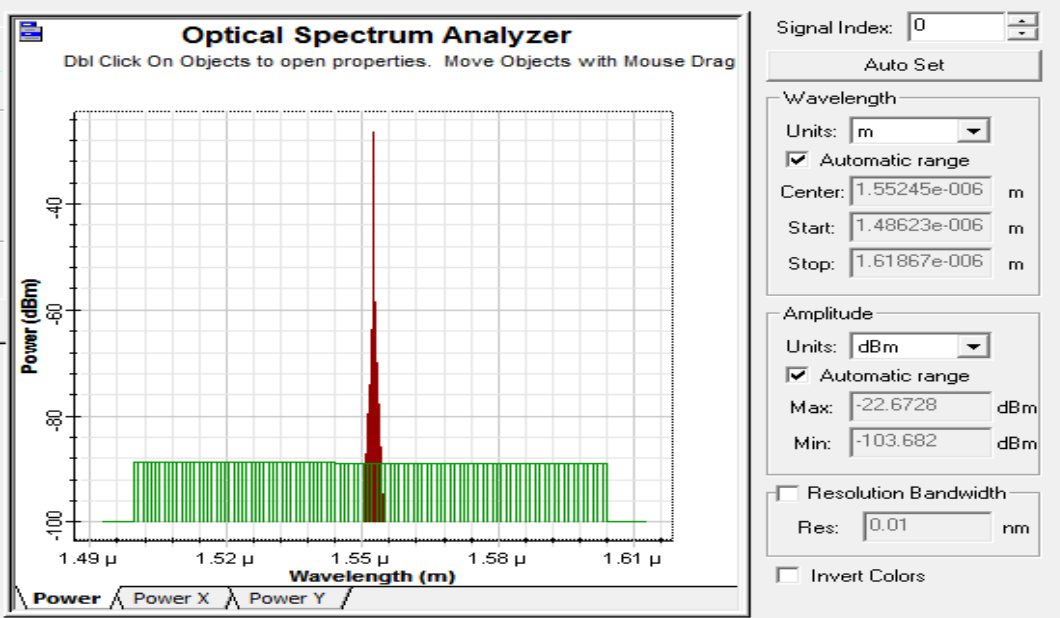

Figure 5. Max. signal power and min. noise power versus wavelength by using NRZ modulation coding scheme based OWC channel (500 km distance) for optical transceiver systems
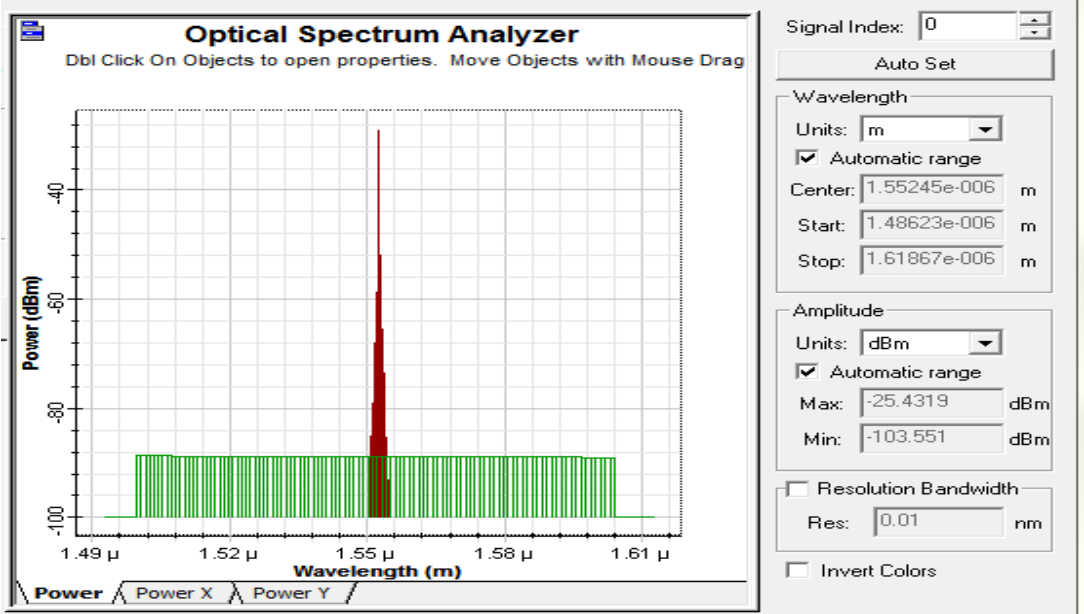

Figure 6. Max. signal power and min. noise power versus wavelength by using RZ modulation coding scheme based OWC channel (500 km distance) for optical transceiver systems
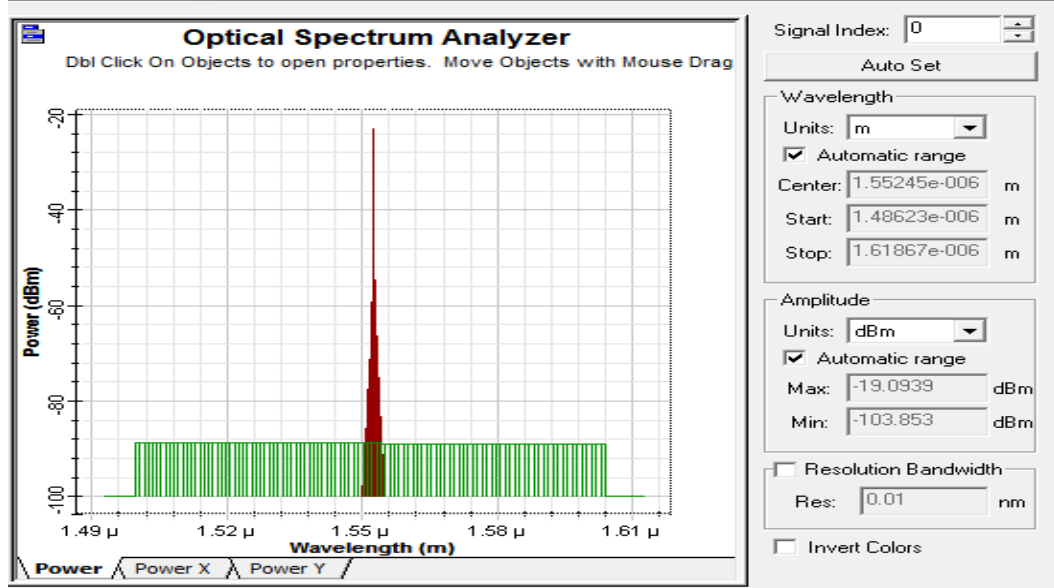

Figure 7. Max. signal power and min. noise power versus wavelength by using On Off keying modulation coding scheme based OWC channel (500 km distance) for optical transceiver systems 
Figures 8-10 show the total power values in Watts and $\mathrm{dBm}$ when using the various modulation coding schemes at a $500-\mathrm{km}$ distance. Figure 8 outlines the total power value was $5.19 \mu \mathrm{W}$ and $-22.849 \mathrm{dBm}$ when using the NRZ modulation coding scheme. For the RZ modulation coding scheme, the total power value was $3.168 \mu \mathrm{W}$ and $-24.992 \mathrm{dBm}$ is shown in Figure 9. The total power value was $8.96 \mu \mathrm{W}$ and -20.477 $\mathrm{dBm}$ when using the On-Off keying modulation coding scheme is clarified in Figure 10.

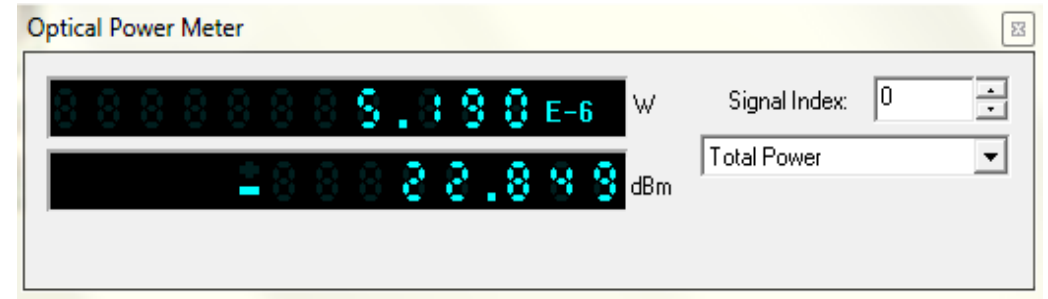

Figure 8. Total power values in Watt and $\mathrm{dBm}$ by using NRZ modulation coding scheme based OWC channel (500 km distance) for optical transceiver systems

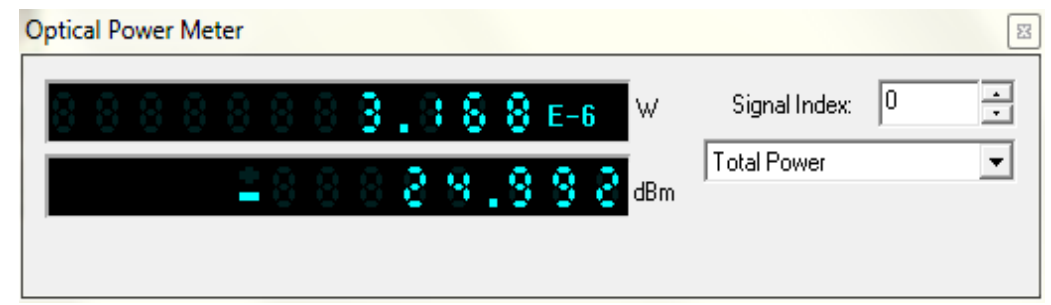

Figure 9. Total power values in Watt and $\mathrm{dBm}$ by using RZ modulation coding scheme based OWC channel (500 km distance) for optical transceiver systems

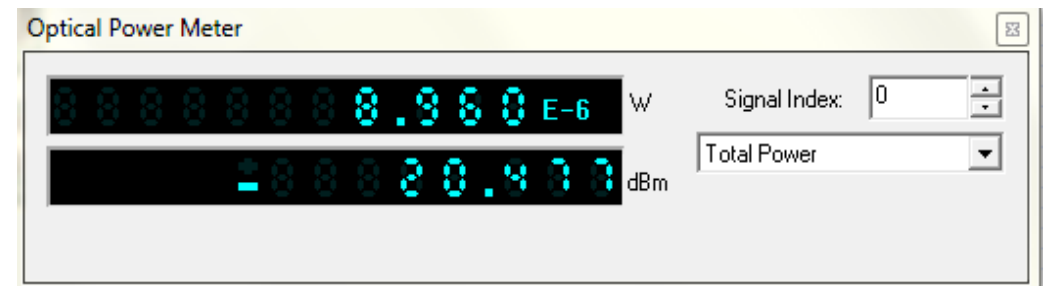

Figure 10. Total power values in Watt and $\mathrm{dBm}$ by using On Off keying modulation coding scheme based OWC channel (500 km distance) for optical transceiver systems

Figure 11 shows the max. Q factor variations with OWC distance for the various modulation coding schemes. The max. Q factorswere 18.18 for the NRZ modulation coding scheme, 13.8 for the RZ modulation coding scheme, and 124.75 for the On-Off keying modulation coding scheme at an OWC distance of 100 $\mathrm{km}$, while the max. Q factorswere 12.26 for the NRZ modulation code scheme, 10.09 for the RZ modulation code scheme, and 20.53 for the On-Off keying modulation code scheme at an OWC distance of $300 \mathrm{~km}$. At an OWC distance of $500 \mathrm{~km}$, themax. Q factorswere 6.45 for the NRZ modulation coding scheme, 5.14 for the RZ modulation coding scheme, and 7.42 for the On-Off keying modulation coding scheme.

Figure 12 shows the received power variations with OWC distance for the various modulation coding schemes. The power received was $129.7 \mu \mathrm{W}$ for the NRZ modulation code scheme, $79.2 \mu \mathrm{W}$ for the RZ modulation code scheme, and $224 \mu \mathrm{W}$ for the On-Off keying modulation code scheme at an OWC distance of $100 \mathrm{~km}$. At an OWC distance of $300 \mathrm{~km}$, the power received was $14.41 \mu \mathrm{W}$ for the NRZ modulation coding scheme, $8.8 \mu \mathrm{W}$ for the RZ modulation coding scheme, and $24.89 \mu \mathrm{W}$ for On-Off keying modulation coding scheme. The power received was $5.19 \mu \mathrm{W}$ for the NRZ modulation coding scheme, 3.169 $\mu \mathrm{W}$ for the $\mathrm{RZ}$ modulation coding scheme, and $8.96 \mu \mathrm{W}$ for the On-Off keying modulation coding scheme at an OWC distance of $500 \mathrm{~km}$. 


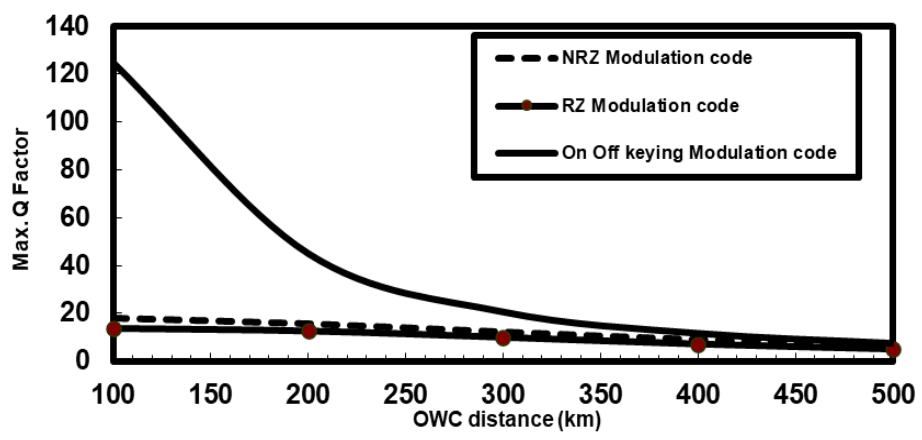

Figure 11. Max. Q factor variations with OWC distance for various modulation coding schemes based OWC channel for optical transceiver systems

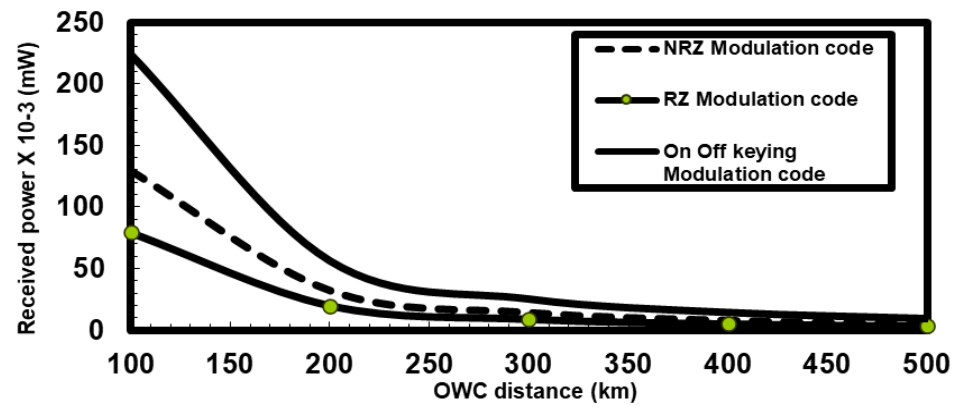

Figure 12. Received power variations with OWC distance for various modulation coding schemes based OWC channel for optical transceiver systems

\section{CONCLUSION}

We simulated the different modulation coding schemes for an optical transceiver system-based OWC channel. The max. Q factors/min BERs for the different modulation schemes were estimated. The max. signal power/min. noise power and the total received power were clarified for the various modulation coding schemes. The study found that On-Off keying resulted ina better max. Q factor/min. BER and max. received power than the other modulation schemes. On-Off keying led to an improvement percentage ratio of $13 \%$ in signal quality and an enhancement percentage ratio of $28 \%$ in max. received power compared with the other modulation schemes at an OWC distance of $500 \mathrm{~km}$.

\section{REFERENCES}

[1] K. K. Wong, et al., "Infrared wireless communication using spread spectrum techniques," IET Digital Library, vol. 147, no. 4, pp. 308-314, 2000.

[2] Ahmed Nabih Zaki Rashed, Mohammed Salah F. Tabbour "The Trade Off Between Different Modulation Schemes for Maximum Long Reach High Data Transmission Capacity Optical Orthogonal Frequency Division Multiplexing (OOFDM)," Wireless Personal Communications Journal, Springer Science, vol. 101, No. 1, pp. 325-337, July 2018.

[3] Ahmed Nabih Zaki Rashed, et al., "Transmission Performance Simulation Study Evaluation for High Speed Radio Over Fiber Communication Systems," Wireless Personal Communications Journal, Springer Science, vol. 103, no. 2, pp. 1765-1779, Nov 2018.

[4] J. R. Barry, J. M. Kahn, E. A. Lee and D. G. Messerschmitt, "High-speed nondirective optical communication for wireless networks," in IEEE Network, vol. 5, no. 6, pp. 44-54, Nov 1991.

[5] D. Kedar and S. Arnon, "Optical wireless communication through fog in the presence of pointing errors," Applied Optics, vol. 42, no. 24, pp. 4946-4954, 2003.

[6] S. J. Urachadaet et al., "Channel modeling for optical wireless communication through dense fog," Journal of Optical Networking, vol. 4, no. 6, pp. 291-299, 2005.

[7] Ahmed Nabih Zaki Rashed, et al., "20 Gb/s Hybrid CWDM/DWDM for Extended Reach Fiber to the Home Network Applications," Proceedings of the National Academy of Sciences, India Section A: Physical Sciences, vol. 89 , no. 4, pp. 653-662, 2019. 
[8] Ahmed Nabih Zaki Rashed, "Comparison between NRZ/RZ Modulation Techniques for Upgrading Long Haul Optical Wireless Communication Systems,” Journal of Optical Communications, 2019.

[9] K. Vinoth Kumar,et al., "Nonlinear Characteristics of Semiconductor Optical Amplifiers for Optical Switching Control Realization of Logic Gates," Journal of Optical Communications, 2019.

[10] J. C. Adriano, et al., "Optical interference produced by artificial light," Wireless Networks, vol. 3, pp. 131-140, May 2, 1997.

[11] J. M. Kahn, W. J. Krause and J. B. Carruthers, "Experimental characterization of non-directed indoor infrared channels," in IEEE Transactions on Communications, vol. 43, no. 2/3/4, pp. 1613-1623, Feb./March/April 1995.

[12] IS Amiri, et al., "Interaction between Optical Sources and Optical Modulators for High-Speed Optical Communication Networks," Journal of Optical Communications, 2019.

[13] IS Amiri,et al., "Effects of Order Super Gaussian Pulses on the Performance of High Data Rate Optical Fiber Channel in the Presence of Self Phase Modulation," Journal of Optical Communications, 2019.

[14] IS Amiri, et al., "Mathematical Model Analysis of Dispersion and Loss in Photonic Crystal Fibers," Journal of Optical Communications, 2019.

[15] IS Amiri, et al., "Basic Functions of Fiber Bragg Grating Effects on the Optical Fiber Systems Performance Efficiency," Journal of Optical Communications, 2019.

[16] IS Amiri, et al., "Nonlinear Effects with Semiconductor Optical Amplifiers," Journal of Optical Communications, 2019.

[17] IS Amiri, et al., "High-Speed Light Sources in High-Speed Optical Passive Local Area Communication Networks," Journal of Optical Communications, 2019.

[18] M. D. Audeh and J. M. Kahn, "Performance evaluation of baseband OOK for wireless indoor infrared LAN's operating at $100 \mathrm{Mb} / \mathrm{s}$," in IEEE Transactions on Communications, vol. 43, no. 6, pp. 2085-2094, June 1995.

[19] S. Yousefi, E. Altman, R. El-Azouzi and M. Fathy, "Analytical Model for Connectivity in Vehicular Ad Hoc Networks," in IEEE Transactions on Vehicular Technology, vol. 57, no. 6, pp. 3341-3356, Nov 2008.

[20] M. Raya and J. P. Hubaux, "Securing Vehicular Ad Hoc Networks," Journal of Computer Security, vol. 15, no. 1, pp. 39-68, 2007.

[21] M. R. Krames et al., "Status and Future of High-Power Light-Emitting Diodes for Solid-State Lighting," in Journal of Display Technology, vol. 3, no. 2, pp. 160-175, June 2007.

[22] B. L. Cole, and Brown, B., "Optimum intensity of red road-traffic signal lights for normal and protanopic observers," Journal of the Optical Society of America, vol. 56, no. 4, pp. 516-522, 1966.

[23] I. Moreno, C.C. Sun, and R. Ivanov, "Far field condition for light emitting diode arrays," Applied Optics, vol. 48, no.6, pp. 1190-1197, 2009.

[24] IS Amiri,et al., "Spatial Continuous Wave Laser and Spatiotemporal VCSEL for High-Speed Long Haul Optical Wireless Communication Channels," Journal of Optical Communications, 2019.

[25] IS Amiri, et al., "Average Power Model of Optical Raman Amplifiers Based on Frequency Spacing and Amplifier Section Stage Optimization," Journal of Optical Communications, 2019.

[26] IS Amiri, et al., "Temperature effects on characteristics and performance of near-infrared wide bandwidth for different avalanche photodiodes structures," Results in Physics, vol. 14, p. 102399, Sep 2019.

[27] IS Amiri, Ahmed NabihZakiRashed, "Simulative study of simple ring resonator-based brewster plate for power system operation stability," Indonesian Journal of Electrical Engineering and Computer Science, vol. 16, no. 2, pp. 1070-1076, Nov 2019.

[28] Ivan Moreno and C. Cherng Sun, "Modeling the radiation pattern of LEDs," Optical Society of America, Optics Express, vol. 16, no. 3, pp. 1808-1819, 2008.

[29] Navin Kumar, et al., "Visible Light Communication Systems Conception and VIDAS," IETE Technical Review, vol. 25, no. 6, pp. 359-367, 2008.

[30] IS Amiri, et al., "Influence of device to device interconnection elements on the system behavior and stability," Indonesian Journal of Electrical Engineering and Computer Science, vol. 18, no. 2, pp. 843-847, 2020.

[31] IS Amiri, et al., "Comparative Simulation Study of Multi Stage Hybrid All Optical Fiber Amplifiers in Optical Communications," Journal of Optical Communications, 2019.

[32] M. Akanegawa, Y. Tanaka and M. Nakagawa, "Basic study on traffic information system using LED traffic lights," in IEEE Transactions on Intelligent Transportation Systems, vol. 2, no. 4, pp. 197-203, Dec 2001.

[33] S. Hidemitsu, et al., "Experimental Investigation of Modulation Method for Visible-Light Communication," IEICE Transsactions on Communication., vol. E89-B, pp. 3393-3400, 2006.

[34] H. Le Minh et al., "High-Speed Visible Light Communications Using Multiple-Resonant Equalization," in IEEE Photonics Technology Letters, vol. 20, no. 14, pp. 1243-1245, July 2008.

[35] M. A. Khalighi and M. Uysal, "Survey on Free Space Optical Communication: A Communication Theory Perspective," in IEEE Communications Surveys \& Tutorials, vol. 16, no. 4, pp. 2231-2258, Fourthquarter 2014.

[36] Yi-LinYu, Shien-KueiLiaw, Hiroki Kishikawa, NobuoGoto, "A simple beam expanded for indoor optical wireless communication with short transmission distance," Optics Communications, vol. 451, pp. 216-219, Nov 2019.

[37] A. T. Hussein and J. M. H. Elmirghani, "10 Gbps Mobile Visible Light Communication System Employing Angle Diversity, Imaging Receivers, and Relay Nodes," Journal of Optical Communications and Networking, vol. 7, no. 8, pp. 718-735, 2015.

[38] S. H. Younus and J. M. H. Elmirghani, "WDM for high-speed indoor visible light communication system," 2017 19th International Conference on Transparent Optical Networks (ICTON), Girona, pp. 1-6, 2017. 
[39] A. T. Hussein, M. T. Alresheedi and J. M. H. Elmirghani, "20 Gb/s Mobile Indoor Visible Light Communication System Employing Beam Steering and Computer Generated Holograms," in Journal of Lightwave Technology, vol. 33, no. 24, pp. 5242-5260, 2015.

[40] A. T. Hussein, M. T. Alresheedi and J. M. H. Elmirghani, "25 Gbps mobile visible light communication system employing fast adaptation techniques," 2016 18th International Conference on Transparent Optical Networks (ICTON), Trento, pp. 1-7, 2016.

[41] S. Dimitrov, S. Sinanovic and H. Haas, "Signal Shaping and Modulation for Optical Wireless Communication," in Journal of Lightwave Technology, vol. 30, no. 9, pp. 1319-1328, 2012.

[42] Z. Wang, Q. Wang, W. Huang, and Z. Xu, "Visible Light Communications: Modulation and Signal Processing," New Jersey: John Wiley \& Sons, Inc., 2017.

[43] Ankita Aggarwal and Gurmeet Kaur, "A Review of Various Modulation Techniques for Indoor Optical Wireless Communication System," Australian Journal of Basic and Applied Sciences, vol. 11, no. 8, pp. 7-11, 2017.

[44] C. T. Manimegalai, S. Gauni, N. Raghavan and T. R. Rao, "Investigations on suitable modulation techniques for visible light communications," 2017 International Conference on Wireless Communications, Signal Processing and Networking (WiSPNET), Chennai, pp. 1818-1822, 2017.

[45] X. Ma, K. Lee, and K. Lee, "Appropriate modulation scheme for visible light communication systems considering illumination," IET Digital Library., vol. 48, no. 18, pp. 1137-1139, 2012. 